\title{
The Method of Internet Sharing for Innovation Experiment in Engineering Education
}

\author{
Zhu Qingjie \\ School of petroleum engineering \\ Changzhou University \\ Changzhou 213016, China \\ qjzhu@cczu.edu.cn
}

\author{
Zhang Bingsheng \\ School of petroleum engineering \\ Changzhou University \\ Changzhou 213016, China \\ 2104117992@qq.com
}

\begin{abstract}
As the progress of innovation experiment in engineering education, especially open innovation experiment, it is urgently needed to investigate the internet sharing method. Through Technical performance analyzing of Web GIS, the method of network sharing with MapXtreme is discussed, the real time calculating in server and browse of experiment results in client are developed. As an example for safety evaluation, system structure and MapXtreme resolution of internet sharing for innovation experiment is discussed. The method of Multi-Criteria Evaluation (MCE) for safety evaluation is introduced; calculating method of weights in MCE method is analyzed as basis of Analytical Hierarchy Program (AHP). System application of weights input and calculating results browse is developed with JavaScript and VBScript as Active Server Page (ASP). Finally, the internet sharing method is analyzed and some advice is proposed.
\end{abstract}

Keywords- Internet sharing; Innovation experiment; Engineering education; WebGIS

\section{INTRODUCTION}

As the informatization progress of engineering education, especially the need of open innovation experiment, it is urgently to investigate the internet sharing method. The construction of web-based education and knowledge sharing environment has been developed quickly, and it is an unprecedented challenge to the experiment design with personal learning environment ${ }^{[1]}$. Many kinds of services can be provided for any users in the world by Internet, such as web-based spatial analysis, maps display, user access, etc. In internet environment, WebGIS technique, such as MapXtreme, can be integrated with web, and GIS analysis, such as MCE evaluation or decision making, can be work out. WebGIS has been applied in many fields, its application in engineering education, will changes the manner of engineering education, especially, innovation experiment design ${ }^{[2-4]}$.

About the evaluation method for engineering safety evaluation, GIS-based multi criteria evaluation (MCE) is a relatively new method. Two types of multi-criteria method are including in GIS. One is Boolean overlay method that is as basis of Boolean operation of intersection and union. Another method, represent as weighted linear combination (WLC), is more important and popular. In weighted linear combination, evaluation criteria are sequential standardized, and weights can be calculated based on Analytic Hierarchy
Process (AHP) ${ }^{[6-9]}$. Due to the advantages of AHP, it has been combined with GIS software and Multi-Criteria Evaluation (MCE) method [10-16]. For example, its combination with IDRISI makes the calculation of factor weights in IDRISI becomes very convenient, where the module WEIGHT utilizes one by one comparison method to help users to construct a comparison matrix. Finally, weights and a consistency ratio are calculated, which is the foundation of MCE safety evaluation.

Internet sharing method and weights calculating method based on AHP for innovation experiments in safety evaluation are discussed in this paper. An example application is practiced and analyzed, comprehension ability for innovation experiment design of college students is improved, and some advice for internet sharing in engineering education is proposed.

\section{INTERNET SHARING METHOD}

Main methods for WebGIS are CGI, plug-in of GIS, Java, ASP, and ActiveX in browser. CGI is the acronym of Common Gateway Interface, and it is a method to integrate gateway scripts into programs. Therefore, it is an HTTP servers' agreement. With this method, customers can interact with Web Server, but difficult for GIS analysis. GIS plug-in, which can be downloaded from the server, receive data vector from the server and process it. In this method, most processing work is completed in the client, and the ability of GIS server is limited. ActiveX is similar to the plug-in, in which GIS analysis is work out in the client through GIS data access. The difference between plug-in and ActiveX control is that ActiveX control is in fact a dynamic link library module.

ASP is a server side technology. Pictures or other content can be dynamic displayed in web pages. This method is easy to learn and developed raptly. All works is work out in the server side, and the ability of GIS server is full used. With this method, HTML page can be created for users, HTML page is combined with dynamic server module and scripts technology.

In this article, ASP method and ActiveX control are combined based on MapXtreme. Through system application development, the input parameters table and evaluation results are displayed to customer as asp web page. System application program are developed by VBScript and JavaScript language in ASP environment. Real-time calculation and evaluation in GIS server, real-time display in 
web server, real-time browse and parameters input in client are achieved.

\section{A. Internet sharing system strucutre}

A three layers model based on $\mathrm{B} / \mathrm{S}$ (Browser/Server) is created for this system, which is shown as figure 1 . The three layers are web browser for users, Web server and GIS Server (MapXtreme). GIS analysis and map process are achieved in GIS server, data transmitted between GIS server and web server based HTTP, parameters input table and results display are controlled by web server. Customers interact with GIS server through web server. Input data management and spatial data analysis are operated by customers with browser. Most works of GIS analysis, such as decision making, spatial variability analysis and weights calculation, are achieved in the server side.

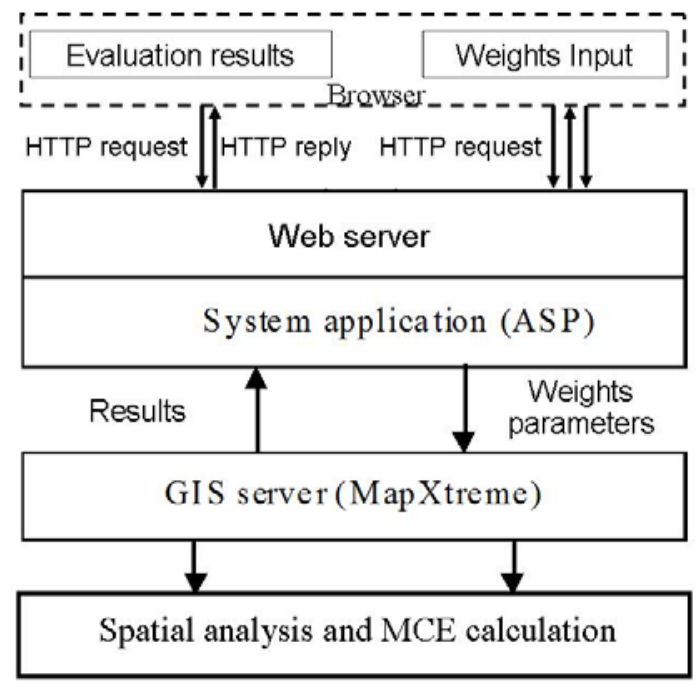

Figure 1. Internet sharing System for Innovation Experiment

\section{B. System application development}

Through the development of system application, many performances of the system, such as pan, zoom in and zoom out, are worked out. For example, the main system application, such as ASP files Edser.asp, is wrote as,

Edser.asp:

"EDserConstants.asp”

$<$ SCRIPT

LANGUAGE="VBScript"

RUNAT="Server">

Function GetServerGroup Name ()

GetServerGroup Name = cEdServerGroup Name

End Function

Function Restore ()

Dim Result

Dim objmaps

On Error Resume Next

Set objmaps $=$ Getmapengine()

If (Strcomp (Request.server variables (cRequestmethod), cPost, vbTextcompare) =0) Then

Dim objmapxst

Result $=$ Createmapxst ()
Set objmapxst $=$ Getmapxst ()

objmapxstate. Stream = Gethtmlformfield

(cCdPreviousmapst)

objmapxst.Restore objmaps

Else

Result $=$ Destroymapxst ()

Result $=$ Setmapsize(Getmapwid(), Getmaph()

Result=Setmapcenter and zoom

(cCdefaultcenterc,cCddefaultcentery, cCddefault zoom)

Result $=$ Setlayerper(objmaps. layers (cCdbd), True, True, False)

Result = Setllayerper(objmaps.layers (cCdwell), True, True, True)

Result $=$ Setlayerper (objmaps. layers (cCdleg), True, False, False)

Result = Setlayerper (objmaps. layers (cCdnub), True, False, True)

Result $=$ Setlayerper (objmaps.layers(cCdEdser), True, True, True)

End If

Restorestate $=$ True

End Function

$</$ SCRIPT $>$.

\section{EVALUATION METHOD}

During safety evaluation, every factor is treated as layer, and weights can be calculated by AHP method. According to the advantage of AHP, AHP provides an effective evaluation method for safety evaluation, especially weights calculation. As usually, or very common cases, weights can be calculated by a simple method that is to divide 1.0 among all influence factors. In fact, there are more than 3 numbers of influence factors, this make weights calculation as a whole set very very difficult. During the safety evaluation procedure for many engineering problems with WLC method as basis of GIS environment, the sum of all the weights must be one. In AHP method, the principal eigenvector can represent calculating weights, and the principal eigenvector comes from the pair wise comparisons for any two factors in all influence factors, and that is shown as a square reciprocal matrix. The relative importance of any two factors can be treating as the comparisons indexes, which determine the situation of engineering safety. Generally, we can use a 9 or 7 points' scale. For example, in 9 points' scale, 7 means very strongly important for one to the compare factor. If the inverse were the case, one would set $1 / 7$. And the like, we can deduce the rest number from this.

In WLC method, weights are an eigenvector. For example, in comparison matrix $\mathrm{A}$, the maximum latent root is writing as $\lambda_{\max }, \mathrm{W}$ represents eigenvector, and weights can be obtained from the calculation of eigenvector $W$, as following,

$$
A W=\lambda_{\max } W
$$

The Eigenvector is calculated as, 


$$
\bar{a}_{i j}=\frac{a_{i j}}{\sum_{k=1}^{n} a_{k j}} \quad i, j=1,2, \cdots, n
$$

Plus every rows,

$$
\bar{W}_{i}=\sum_{j=1}^{n} \bar{a}_{i j} \quad i, j=1,2, \cdots, n
$$

Through standardized, Vector $\bar{W}=\left[\bar{W}_{1}, \bar{W}_{2}, \cdots, \bar{W}_{n}\right]^{T}$ is obtained from,

$$
W_{i}=\frac{\bar{W}_{i}}{\sum_{j=1}^{n} \bar{W}_{j}} \quad i, j=1,2,3, \cdots, n
$$

The weights can be obtained from eigenvector that equal to $W=\left[W_{1}, W_{2}, \cdots, W_{n}\right]^{T}$.

$\lambda_{\max }$ represents the maximum latent root, is calculated as,

$$
\lambda_{\max }=\sum_{i=1}^{n} \frac{(A W)_{i}}{n W_{i}} .
$$

In which, $(A W)_{i}$ means the i-th factor in $A W$.

C.I. is the acronym of consistency index, it is calculated as,

$$
\text { C.I. }=\frac{\lambda_{\max }-n}{n-1}
$$

Consistency ratio (C.R.) is as follows,

$$
\text { C.R. }=\frac{\text { C.I. }}{\text { R.I. }}
$$

In which, R.I. is randomly consistency index.

For a good consistency, the value of consistency ratio should be less than 0.10 .

If the value of consistency ratio is large than 0.10 , it means that we should modify the comparison matrix and to calculate it again. In order to keep good consistency, we need to know the reason where the inconsistencies aroused. Of course, we know the inconsistency comes from the matrix, thus, we can analyzed this from comparison matrix investigation. For example, if we construct a comparison matrix as follow,

$$
A=\left[\begin{array}{ccc}
1 & 2 & 6 \\
1 / 2 & 1 & 4 \\
1 / 6 & 1 / 4 & 1
\end{array}\right]
$$

The weights for each column are achieved by the process that averaging over all columns and make each column sum to 1.0. For example, if the sum of first column is 1.67, all factors in first column divided by 1.67. The calculating results of weights are $0.60,0.30$, and 0.10 . Through repeating this process for each column, then make all column averaged, the weights are obtained. The principal eigenvector are approximately calculated, and the results as equation (9),

$$
w=\left[\begin{array}{lll}
0.588 & 0.322 & 0.090
\end{array}\right]^{T} \quad \lambda=3.010 .
$$

It can be found that the sum of weights with this method is 1.0. Then the weights can be used in safety evaluation.

If we modify equations (8) as,

$$
A=\left[\begin{array}{ccc}
1 & 2 & 6 \\
1 / 2 & 1 & 3 \\
1 / 6 & 1 / 3 & 1
\end{array}\right]
$$

The results of equations (9) are,

$$
w=\left[\begin{array}{lll}
0.6 & 0.3 & 0.1
\end{array}\right]^{T} \quad \lambda=3
$$

Through compare equations (8) with equations (10), the lack of consensus comes from the inconformity of $\mathrm{A} 1, \mathrm{~A} 2$, and $A 3$. Because $A 1 / A 2=2, A 1 / A 3=6$, then should $A 2 / A 3=3$. But in equations (8), $A 2 / A 3=3.010 \neq 3$, this is the reason of lack of consensus.

\section{APPLICATION EXAMPLE}

As an example application, four factors that influence the oil well safety, and there are 120 oil wells in the research area. The purpose of this experiment is to evaluate the oil field safety based on 120 oil wells production data. The relative importance between any two factors is given a significant value in analytical hierarchy program method. The comparison matrix " $\mathrm{A}$ " is constructed as table 1 .

TABLE I. COMPARISON MATRIX

\begin{tabular}{|c|c|c|c|c|}
\hline Factor & F1 & F2 & F3 & F4 \\
\hline F1 & 1 & & & \\
\hline F2 & $1 / 2$ & 1 & & \\
\hline F3 & 4 & 6 & 1 & \\
\hline F4 & $1 / 5$ & $1 / 3$ & $1 / 8$ & 1 \\
\hline
\end{tabular}

As a symmetrical matrix, only the upper or lower triangular is needed to complete. The remaining cells in the upper triangular half of comparison matrix the reciprocals of the lower triangular half. Such as, the rating of F3 to F1 is 
value 4, the relative important rating of F1 to F3 will simply be $1 / 4)$.

With equations (7), an index of consistency is calculated, this is named consistency ratio.

The calculating results of weights for safety evaluation are,

$$
w=0.2077 \quad 0.1164 \quad 0.6248 \quad 0.0511^{T} \quad \text { C.R. }=0.04<0.1
$$

The calculating steps are shown as figure 2. The application safety evaluation result of this experiment is shown as figure 3 .

The experiment design provides several common tools in browser, such as pan, zoom in, and zoom out. User can browse the vector image with mouse action very simply; operations include dragging, zoom and click. Therefore, ASP method is effective for open remote experiment design through internet. All calculation and evaluation are work out by the server. User can do the experiment through browser conveniently.

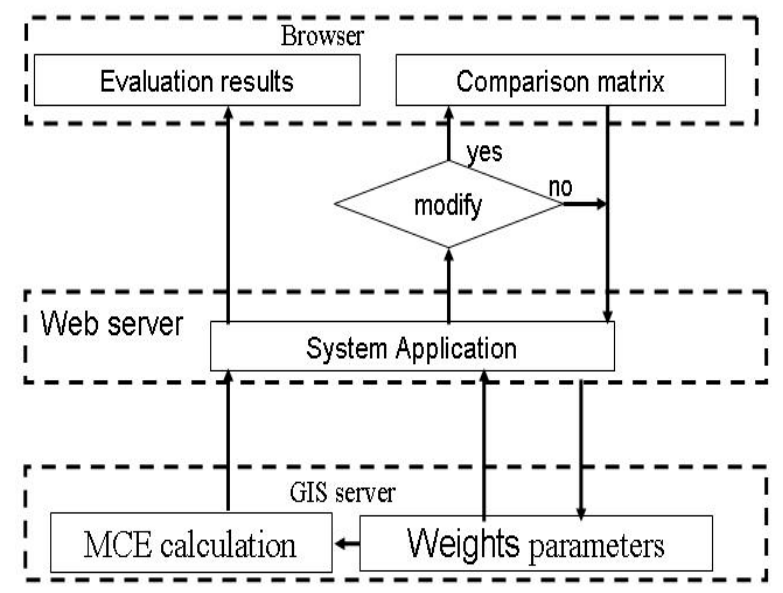

Figure 2. Evaluation process

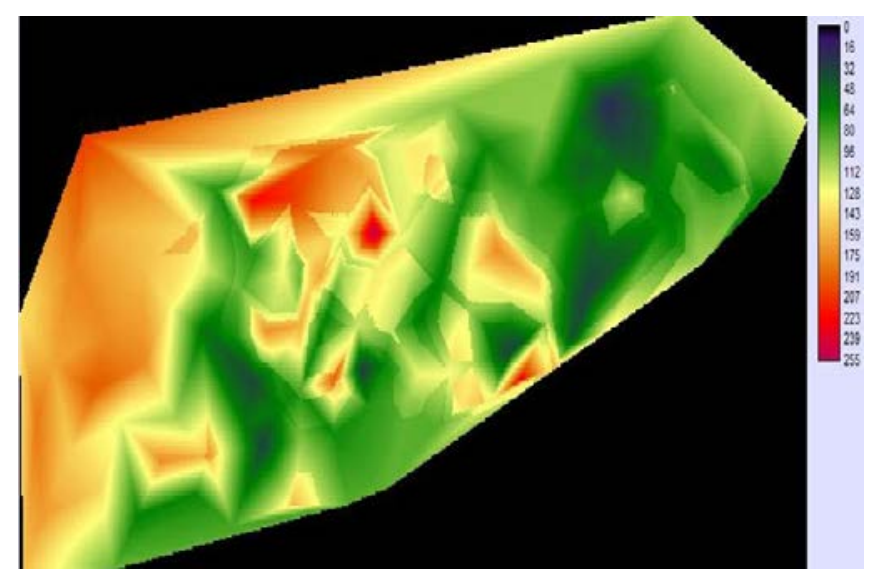

Figure 3. Experiment calculating result

\section{CONCLUSIONS}

MapXtreme is applied to open innovation experiment design, and remote access is achieved through system application development. User can input experiment parameters and obtain experiment results through browser conveniently. With GIS server, MCE method is applied to evaluate the safety of oil well based on production data. Through analysis of AHP method, the relation between comparison matrix and consistency index is investigated. With any change of the pairwise comparisons, the degree of consistency will change. The key of good consistency is to keep the pairwise ratio conformity of all factors.

Therefore, open experiment can be developed for distance education through combing of browser, web server and GIS server.

\section{ACKNOWLEDGMENT}

This work is supported by the Twelfth Five Year Plan Project of Education Science of Jiangsu Province (D/2013/01/130), China-Poland Inter-Governmental Science and Technology Cooperation Project (2012-35-05), and Oversea Scholarship of Jiangsu Province (JS-2013-326).

\section{REFERENCES}

[1] Y. Rui, P. Lili, "Strategies of Knowledge Sharing in Web 2.0 Personal Learning Environment and Their Evaluation," Open Education Research, vol. 21(2),pp.78-88, 2015.

[2] Z., Qingjie, and J. Chen. "MapXtreme Resolution for Internet Publication of Natural Disaster Information in Earthquake Engineering Based on ANN", Systems Engineering Procedia, vol.1,pp.4-9, 2011.

[3] H.L. Yu, L. Wu, Y. Liu, etal., "A Study of integration between GIS and GIS-based model based on Web services”, Journal of Chongqing Jianzhu University,28(4),pp.24-28, 2006.

[4] H. Wang, Z.Q. Wang, C. Li, "Study on the integration frame work of urban planning information system based on Web Services”, ACTA Geodaetica et Cartographica Sinica, vol.35(2), pp.153-159, 2006.

[5] L.A. Vidal, F. Marle, J.C. Bocquet, "Using a Delphi process and the Analytic Hierarchy Process (AHP) to evaluate the complexity of projects”, Expert Systems with Applications, vol.38, pp. 5388-5405, 2011.

[6] T.L. Saaty, J.S. Shang, “An innovative orders-of-magnitude approach to AHP-based mutli-criteria decision making: Prioritizing divergent intangible humane acts”, European Journal of Operational Research, vol.214, pp.703-715, 2011.

[7] Z. Hualin, L. Gang, “Application of analytic hierarchy process to petroleum safety evaluation”, Natural Gas Industry, vol.26(4), pp. 135-137, 2006.

[8] Q. Huiqiong, L. Hualiang, “Application of AHP Model in Teaching Quality Evaluating of the Situation and Policy in Universities", Journal of Central South University (Science and Technology), vol.22(2), pp. 75-80, 2008.

[9] M. Javadiana, H. Shamskooshkia, M. Momenia, “Application of sustainable urban development in environmental suitability analysis of educational land use by using AHP and GIS in Tehran”, Procedia Engineering, vol.21, pp.72-80, 2011.

[10] S. Chakhar, J.M. Martel, "Enhancing Geographical Information System Capabilities with Multi-Criteria Evaluation Functions”, Journal of Geographic Information and Decision Analysis, Vol. 7(2), pp.47-71, 2003. 
[11] Z. Qingjie, S. Youpo, D. Wu, "Risk assessment of land-use suitability and application to Tangshan City", International Journal of Environment and Pollution, vol.42(10), pp.330-343, Oct. 2010.

[12] Z. Qingjie, B. Hejmanowska, "Analysis of GIS-based spatial variability and risk assessment", Journal of Chemical and Pharmaceutical Research, vol.5(9),pp.372-380, Sep. 2013.

[13] J. Taveau, "Risk Assessment and Land-use Planning Regulations in France Following the AZF Disaster", Journal of Loss Prevention in the Process Industries, Vol. 23, pp.813-823, 2010.
[14] J. Malczewski, “Ordered Weighted Averaging with Fuzzy Quantifiers: GIS-based Multicriteria Evaluation for Land-use Suitability Analysis”, International Journal of Applied Earth Observation and Geoinformation, Vol. 8(4), pp.270-277, 2006.

[15] X. Weibin, Z. Qingjie, J. Haibo, "The Comprehensive Evaluation Method Based on GIS of Oil well Casing Damage”, Advanced Materials Research, vol. 807-809(9),pp.2595-2601, 2013.

[16] Y.H. Guo, B.G. Wang, "Two Types of New Neural Networks and Their Applications to Safety Assessment”, China Safety Science Journal, vol.18(7), pp.29-33, June 2008. 